Wright State University

CORE Scholar

Mechanical and Materials Engineering Faculty

Publications

Mechanical and Materials Engineering

$1-17-2017$

\title{
Identifying Two Regimes of Slip of Simple Fluids Over Smooth Surfaces with Weak and Strong Wall-Fluid Interaction Energies
}

Haibao Hu

Luyao Bao

Nikolai V. Priezjev

Wright State University - Main Campus, nikolai.priezjev@wright.edu

Kai Luo

Follow this and additional works at: https://corescholar.libraries.wright.edu/mme

Part of the Materials Science and Engineering Commons, and the Mechanical Engineering Commons

\section{Repository Citation}

The download link is the publisher version, it is available following the embargo period in the publisher's condition.

This Article is brought to you for free and open access by the Mechanical and Materials Engineering at CORE Scholar. It has been accepted for inclusion in Mechanical and Materials Engineering Faculty Publications by an authorized administrator of CORE Scholar. For more information, please contact library-corescholar@wright.edu. 


\title{
Identifying two regimes of slip of simple fluids over smooth surfaces with weak and strong wall-fluid interaction energies
}

\author{
Haibao Hu, ${ }^{1, a), b)}$ Luyao Bao, $^{1, b)}$ Nikolai V. Priezjev, ${ }^{2, a)}$ and Kai Luo ${ }^{1}$ \\ ${ }^{1}$ School of Marine Science and Technology, Northwestern Polytechnical University, Xi'an, Shaanxi 710072, \\ People's Republic of China \\ ${ }^{2}$ Department of Mechanical and Materials Engineering, Wright State University, Dayton, Ohio 45435, USA
}

(Received 26 August 2016; accepted 22 December 2016; published online 17 January 2017)

\begin{abstract}
The slip behavior of simple fluids over atomically smooth surfaces was investigated in a wide range of wall-fluid interaction (WFI) energies at low shear rates using non-equilibrium molecular dynamics simulations. The relationship between slip and WFI shows two regimes (the strong-WFI and weakWFI regimes): as WFI decreases, the slip length increases in the strong-WFI regime and decreases in the weak-WFI regime. The critical value of WFI energy that separates these regimes increases with temperature, but it remains unaffected by the driving force. The mechanism of slip was analyzed by examining the density-weighted average energy barrier $(\overline{\Delta E})$ encountered by fluid atoms in the first fluid layer (FFL) during their hopping between minima of the surface potential. We demonstrated that the relationship between slip and WFI can be rationalized by considering the effect of the fluid density distribution in the FFL on $\overline{\Delta E}$ as a function of the WFI energy. Moreover, the dependence of the slip length on WFI and temperature is well correlated with the exponential factor $\exp \left(-\overline{\Delta E} /\left(k_{\mathrm{B}} T\right)\right)$, which also determines the critical value of WFI between the strong-WFI and weak-WFI regimes. Published by AIP Publishing. [http://dx.doi.org/10.1063/1.4973640]
\end{abstract}

\section{INTRODUCTION}

For centuries, the no-slip boundary condition (BC) has been successfully used in analyzing macroscale liquid flows because it can reproduce the flow characteristics measured in experiments. In the past few decades, experiments ${ }^{1-5}$ and computer simulations ${ }^{6-11}$ demonstrated that a liquid can slip relative to the solid boundary. The most common way to measure slip is by using the concept of the slip length introduced by Navier, which states that the slip length is defined as an extrapolated distance where the fluid velocity profile vanishes. ${ }^{3}$ Typical values reported in experiments for the slip length are of the order of few nanometers leading to a conclusion that slip can be safely ignored in most macroscale flow systems. ${ }^{12}$ However, even a small amount of slip can be important in small-scale flows, which are present, for example, in nanofluidic devices. A critical technological problem of these devices is the high flow resistance owing to the extremely small channel dimensions. ${ }^{11,13}$ By definition, the friction coefficient $(k)$ of the wall/fluid interface is inversely related to the slip length: $k=\mu / L_{S}$, where $\mu$ is the viscosity of fluid. ${ }^{14}$ Therefore, slippage can provide a promising way to reduce the high resistance in nanoflows.

To properly describe and manipulate liquid flow in a highly confined geometry, such as flows in nanochannels, the mechanism of slip needs to be understood at the atomic level. During the past decades, molecular dynamics (MD) simulations were extensively used to study the correlation between

\footnotetext{
a) Authors to whom correspondence should be addressed. Electronic addresses: huhaibao@nwpu.edu.cn and nikolai.priezjev@wright.edu

b) Haibao Hu and Luyao Bao have contributed equally to this work.
}

slip and various interfacial parameters ${ }^{6-8,11,13-24}$ since this method can provide atomistic resolution of velocity profiles and dynamics of fluid atoms in contact with solid walls. The dominant factors influencing liquid slip over atomically smooth surfaces are the wall-fluid interaction (WFI) energy, shear rate, commensurability between liquid and solid structures, and thermodynamics parameters (temperature, pressure, etc.).

The inverse correlation between slip and WFI has been repeatedly observed in many previous MD studies, ${ }^{7,17,20,22}$ which is consistent with the intuition that weak WFI reduces the friction between a fluid and a smooth solid wall. Along with decreasing WFI, the slip length was shown to be correlated inversely with the degree of order induced in the first fluid layer (FFL) by the periodic surface potential. ${ }^{10,14,18-20}$ Increasing shear rate and/or incommensurability between liquid and solid structures reduces the order within FFL, hence slip is enhanced. ${ }^{10,18}$

The slip model ${ }^{10,14}$ based on the fluid structure shows that the slip length is directly related to the main peak value of the in-plane structure factor of a liquid in the FFL. The inplane fluid structure factor is defined as $S(\mathbf{k})=1 / N_{l}\left|\sum_{j} e^{i \mathbf{k} \cdot \mathbf{r}_{j}}\right|^{2}$, where $\mathbf{r}_{j}=\left(x_{j}, y_{j}\right)$ is the two-dimensional position vector of the $j$ th atom and the sum is taken over $N_{l}$ atoms within the FFL. ${ }^{17}$ Furthermore, Martini et al. ${ }^{13,16}$ studied the so-called "defect-slip" mechanism by investigating the hopping dynamics of fluid atoms in the FFL at low shear rates. Using a similar concept, Wang and Zhao ${ }^{25}$ proposed an explicit quantitative expression for the slip velocity. According to their work, the magnitude of slip velocity depends on the average shear stress, the temperature, and the energy barrier encountered by atoms during hopping. Decreasing WFI leads to a 
reduction in the energy barrier, and, consequently, an increase of slip because atoms can hop more easily. Thus the negative correlation between slip and WFI is recovered.

The mechanism of slip proposed by Martini et al. ${ }^{13,16}$ and Wang and $\mathrm{Zhao}^{25}$ predicts a monotonic relation between slip and WFI, which is consistent with previous MD simulation results. ${ }^{7,17,20,22,26-28}$ However, when WFI is less than a critical value (about six percent of the interaction energy between fluid atoms), Liu and $\mathrm{Li}^{29}$ observed a positive correlation between the WFI energy and the fluid mass flux, which is another way to measure the degree of slip. Most of the previous MD studies that reported inverse relation between slip and WFI were carried out with the WFI energy greater than ten percent of the interaction energy between fluid atoms. ${ }^{7,17,20,22,26-28}$ Therefore, the slip length appears to exhibit two distinct regimes depending on WFI, which has not been studied systematically. Moreover, the mechanism of slip at weak WFI still remains unclear.

The present work provides a detailed analysis of the slip behavior in a wide range of WFI and explores the mechanism of slip at weak WFI using non-equilibrium MD simulations. Our numerical results demonstrate that the negative correlation between the slip length and WFI is consistently followed by the positive correlation with decreasing WFI for various flow conditions. The critical value of WFI between these slip regimes increases with temperature but it is independent of shear rate. We will show that the two-regime relationship between slip and WFI is a result of a competition between a reduced WFI energy and the variation of fluid density distribution in the FFL.

The paper is organized as follows. The details of MD simulations are presented in the Sec. II. Results for the slip length in a wide range of WFI at different shear rates and temperatures are presented in Sec. III A. The mechanism slip is discussed in Sec. III B. The summary is given in the last section.

\section{MD SIMULATION MODEL}

The geometry of simulation is a slab of fluid confined between two face-centered-cubic (FCC) (001) solid walls (see Fig. 1). The interaction between fluid atoms or fluid and wall atoms is both modeled using Lennard-Jones (LJ/126) potential

$$
E_{i j}=4 \varepsilon_{\alpha \beta}\left[\left(\frac{\sigma_{\alpha \beta}}{r_{i j}}\right)^{12}-\left(\frac{\sigma_{\alpha \beta}}{r_{i j}}\right)^{6}\right],
$$

where $E_{i j}$ is the energy between atoms $i$ and $j, \varepsilon$ and $\sigma$ are the characteristic energy and length of the potential, respectively. Here $\alpha$ and $\beta$ are types of atoms $i$ and $j$. The indices $\alpha \beta$ denote either fluid-fluid (FF) or wall-fluid (WF), which corresponds to the interaction between fluid atoms or fluid and wall atoms. The LJ units defined by the intrinsic properties of the fluid: $m_{\mathrm{FF}}, \varepsilon_{\mathrm{FF}}$, and $\sigma_{\mathrm{FF}}$ are used to express all the physical units and the subscripts will be ignored, if these quantities are treated as units. A cutoff distance $\left(r_{\mathrm{c}}=2.5 \sigma\right)$ of the LJ potential was adopted to improve the computational efficiency. The dimensions of the simulation domain in the $x$ and $z$ directions are $58.14 \sigma$ and $28.21 \sigma$, respectively. The height of the channel and the density of the fluid are $29.36 \sigma$ and

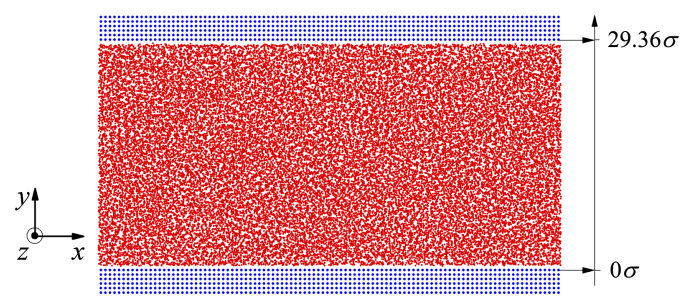

FIG. 1. The front view of the simulation domain. The symbols $(\bullet$, blue $)$ and $(\bullet$, red) denote the solid and fluid atoms. The fluid phase consists of 39168 atoms and each wall contains 15300 atoms. The vertical axis $(y)$ shows $y=0 \sigma$ and the channel height.

$0.813 \sigma^{-3}$, respectively. Both solid walls shown in Fig. 1 were constructed using the face-centered-cubic lattice constant of $1.15 \sigma$. The rigid wall model was adopted where wall atoms were fixed to their lattice sites. The wall density is $4.35 \sigma^{-3}$ and the density ratio between a fluid and a solid corresponds to a high degree of incommensurability between the adjacent fluid and the wall structure. ${ }^{10}$ The thickness of the walls was set to be greater than the cutoff distance.

The planar Poiseuille flow was generated by a constant external force $F_{x}$ in the $+x$ direction, which was added to the equation of motion for each fluid atom. ${ }^{17,18} \mathrm{~A}$ biased Nosé-Hoover thermostat ${ }^{30}$ is applied to the fluid phase to maintain the temperature in the system. While computing the temperature, the kinetic energy is calculated after subtracting out a spatially-averaged center-of-mass velocity field of the $x$ component of fluid atoms. This is done by dividing the simulation domain into 100 bins parallel to the solid walls. All simulations are carried out using the LAMMPS MD code ${ }^{31}$ with a time step of $\Delta t=0.002 \tau$, where $\tau=\left(m \sigma^{2} / \varepsilon\right)^{1 / 2}$. The damping parameter used in the Nosé-Hoover thermostat is $0.2 \tau$. The force $F_{x}$ was applied to each fluid atom after equilibration of the system during $10^{5}$ time steps, and additional $10^{6}$ time steps were used to reach the steady Poiseuille flow.

The fluid density and velocity profiles were averaged within slices of thickness $\Delta y=0.02 \sigma$ for additional $2 \times 10^{6}$ time steps. The slip length $\left(L_{\mathrm{S}}\right)$ was computed using the Navier slip model: $L_{\mathrm{S}}=v_{\mathrm{S}} / \dot{\gamma}$, where $v_{\mathrm{S}}$ and $\dot{\gamma}$ are the slip velocity and shear rate at the wall-fluid interface, which is set at the position of the bottom layer for the upper wall and the top layer for the lower wall. The parameters $v_{\mathrm{S}}$ and $\dot{\gamma}$ were calculated by extrapolating the parabolic fit of velocity profiles with respect to the wall-fluid interface. The velocity profiles used for fitting were computed from $5.01 \sigma$ to $24.99 \sigma$ to exclude the portions near both walls. ${ }^{10,11,19,32}$

\section{RESULTS AND DISCUSSION}

\section{A. Slip length versus wall-fluid interaction energy}

The variation of the slip length $\left(L_{\mathrm{S}}\right)$ versus WFI energy $\left(\varepsilon_{\mathrm{WF}}\right)$ for different temperatures $(T)$ and driving forces $\left(F_{x}\right)$ is presented in Fig. 2. With decreasing $\varepsilon_{\mathrm{WF}}$, the slip length exhibits two distinct regimes, namely, $L_{\mathrm{S}}$ first increases and then decreases when $\varepsilon_{\mathrm{WF}}$ becomes smaller than a critical value $\varepsilon_{L s}^{\max }$. At a given $T$ and $F_{x}, \varepsilon_{L s}^{\max }$ is defined as the WFI energy, where $L_{\mathrm{S}}$ as a function of $\varepsilon_{\mathrm{WF}}$ reaches maximum. This relationship between $L_{\mathrm{S}}$ and $\varepsilon_{\mathrm{WF}}$ holds at different $T$ and $F_{x}$. In what follows, we call the negative correlation between $L_{\mathrm{S}}$ and 

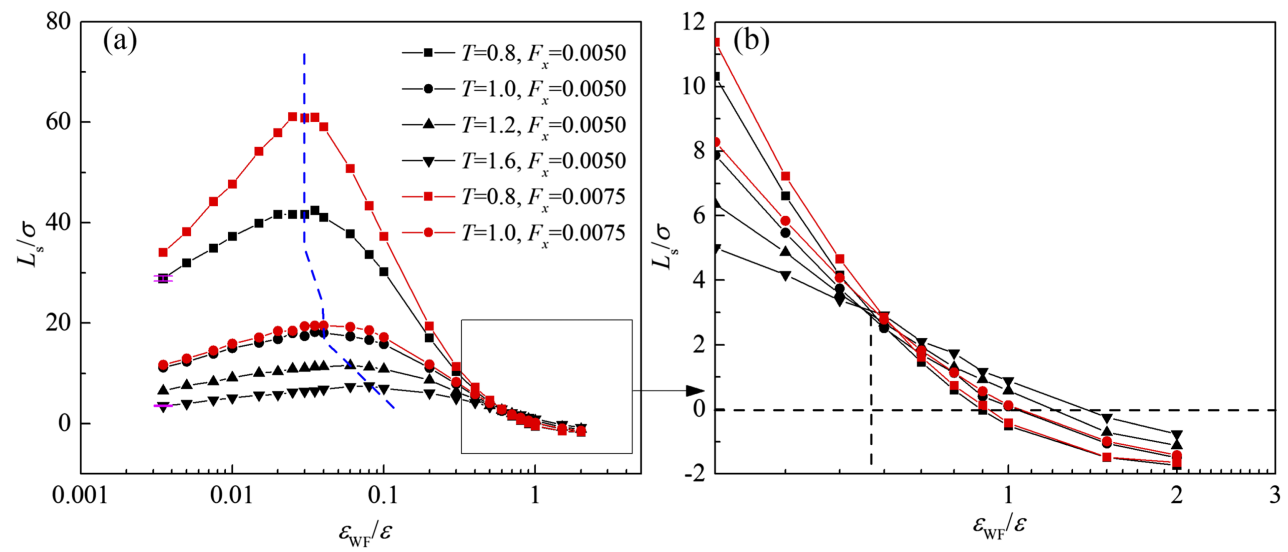

FIG. 2. The slip length $\left(L_{\mathrm{S}}\right)$ versus the wall-fluid interaction (WFI) energy $\varepsilon_{\mathrm{WF}}$ for different temperatures $(T)$ and driving forces $\left(F_{x}\right)$. The units of $T$ and $F_{x}$ are $\varepsilon / k_{\mathrm{B}}$ and $\varepsilon / \sigma$. The right panel represents the enlarged view of $L_{\mathrm{S}}-\varepsilon_{\mathrm{WF}}$ in the strong-WFI slip regime. The blue dashed line in the left panel is a guide to the eye in order to distinguish the strong-WFI and weak-WFI slip regimes. The vertical dashed line in the right panel indicates the intersection point and the value of $\varepsilon_{\mathrm{CR}}^{\mathrm{I}}$. The typical error bars were computed from five independent simulations with different initial atomic momenta distributions. Each simulation was carried out in the same way as described in Section II except that $10^{5} \mathrm{MD}$ steps were used to average the fluid density and velocity profiles.

$\varepsilon_{\mathrm{WF}}$ as the strong-WFI slip regime, and, correspondingly, the positive correlation between $L_{\mathrm{S}}$ and $\varepsilon_{\mathrm{WF}}$ as the weak-WFI slip regime. The critical value of WFI energy between strong- and weak-WFI slip regimes, $\varepsilon_{L s}^{\max }$, increases as $T$ is varied from $0.8 \varepsilon / k_{\mathrm{B}}$ to $1.6 \varepsilon / k_{\mathrm{B}}$ when $F_{x}=0.005 \varepsilon / \sigma$, while $\varepsilon_{L \mathrm{~s}}^{\max }$ remains unchanged as $F_{x}$ is varied from $0.0025 \varepsilon / \sigma$ to $0.01 \varepsilon / \sigma$ for $T=1.0 \varepsilon / k_{\mathrm{B}}$ [see Fig. 3]. A similar dependence of $\varepsilon_{L \mathrm{~s}}^{\max }$ on $T$ also holds for $F_{x}=0.0075 \varepsilon / \sigma$. These results demonstrate that $\varepsilon_{L \mathrm{~s}}^{\max }$ is positively correlated with $T$ and independent of $F_{x}$.

It can be observed in Fig. 2(b), that $L_{\mathrm{S}}-\varepsilon_{\mathrm{wF}}$ curves intersect each other at different $T$. We denoted the intersection point as $\varepsilon_{\mathrm{CR}}^{\mathrm{I}}$. Furthermore, $T$ affects the slip length in two opposite ways, when $\varepsilon_{\mathrm{WF}}$ is either larger or smaller than $\varepsilon_{\mathrm{CR}}^{\mathrm{I}}$. When $\varepsilon_{\mathrm{WF}}>\varepsilon_{\mathrm{CR}}^{\mathrm{I}}, L_{\mathrm{S}}$ increases with $T$, while at $\varepsilon_{\mathrm{WF}}<\varepsilon_{\mathrm{CR}}^{\mathrm{I}}, L_{\mathrm{S}}$

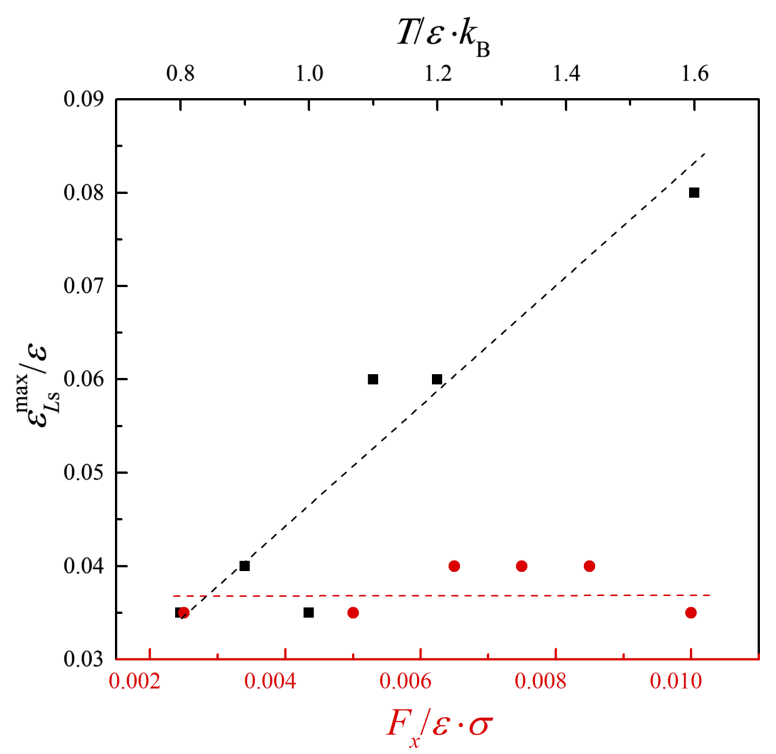

FIG. 3. Variation of $\varepsilon_{L s}^{\max }$ as a function of temperature and driving force. The parameter $\varepsilon_{L \mathrm{~s}}^{\max }$ denotes the wall-fluid interaction energy where the slip length reaches maximum. The symbols (black filled square) and (red filled circle) indicate $\varepsilon_{L \mathrm{~s}}^{\max }$ versus $T$ and $F_{x}$, respectively. $F_{x}$ is varied from 0.0025 to $0.01 \varepsilon / \sigma$ at $T=1.0 \varepsilon / k_{B}$, and $T$ is changed from 0.8 to $1.6 \varepsilon / k_{\mathrm{B}}$ at $F_{x}=0.005 \varepsilon / \sigma$. The black and red dashed lines show trends of $\varepsilon_{L \mathrm{~s}}^{\max }$ as a function of temperature and driving force, respectively. decreases with $T$. With increasing $F_{x}, L_{\mathrm{S}}$ increases in the whole range of $\varepsilon_{\mathrm{WF}}$ at the same $T$, which is consistent with published results. ${ }^{18}$ When $\varepsilon_{\mathrm{WF}}$ becomes sufficiently large, several fluid layers near the wall were locked to the substrate and thus they can be regarded as immobile epitaxial layers. ${ }^{10}$ In this case, the slip length is negative since by definition it was calculated by extrapolating the parabolic fit of velocity profile with respect to the wall-fluid interface. Higher degree of locking of fluid layers leads to more negative slip. Therefore, the negative slip length reflects the degree of locking of fluid layers near the walls. In other words, a small value of the negative slip length means that fluid flows over the locked fluid layers but it does not imply that the flow is in the opposite direction (there is no backflow in this geometry). With decreasing $\varepsilon_{\mathrm{wF}}$, the slip length increases from negative to positive. We denoted $\varepsilon_{\mathrm{CR}}^{0}$ as the critical value of $\varepsilon_{\mathrm{WF}}$ between negative and positive values of the slip length, and it can be seen in Fig. 2(b) that $\varepsilon_{\mathrm{CR}}^{0}$ increases with $T$ and $F_{x}$. We note that the positive correlation between $\varepsilon_{\mathrm{CR}}^{0}$ and $F_{x}$ is not significant owing to the small increment of $F_{x}$ from $0.005 \varepsilon / \sigma$ to $0.0075 \varepsilon / \sigma$.

\section{B. Analysis of the slip behavior in the weak and strong WFI regimes}

Many previous studies ${ }^{13,14,16,18,20,25,32}$ have demonstrated that the first fluid layer (FFL) plays a crucial role in describing slip phenomena. The influence of the remaining layers on slip needs to be considered only at high shear rates, ${ }^{25}$ which is not the case in our study. Martini et al. ${ }^{13,16}$ and Wang and Zhao. ${ }^{25}$ suggested that the slip velocity is proportional to the difference between hopping rates of FFL fluid atoms from one equilibrium site to another along and opposite to the flow direction. Fluid atoms in the FFL experience a corrugated surface potential determined by WFI, lattice structure, and thermal roughness. ${ }^{14,32}$ In our study, we focused on the influence of WFI. As shown in Fig. 4(a), the surface potential consists of local maxima and minima periodically distributed at the wall lattice plane.

Atoms in the FFL prefer to occupy the local minima of the surface potential, while some of these atoms occasionally 


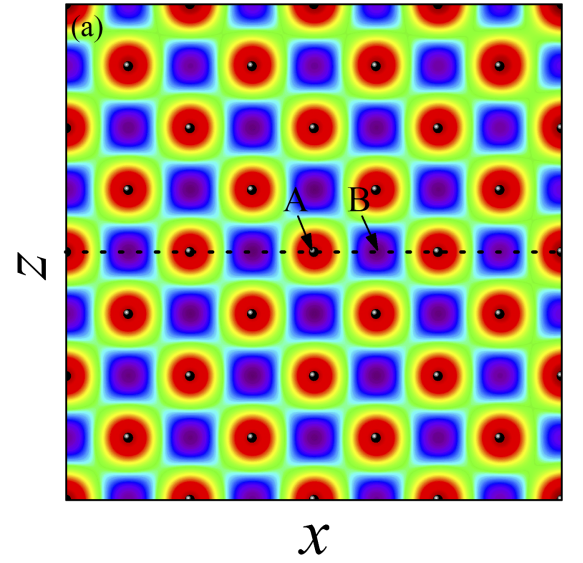

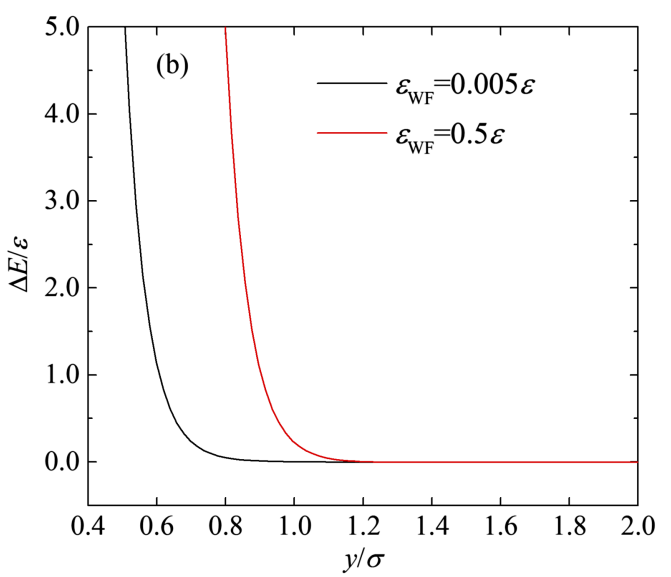

FIG. 4. (a) An example of the potential surface of the top wall layer (black spheres) in a plane parallel to the wall for $\varepsilon_{\mathrm{WF}}=0.5 \varepsilon$. The potential is computed by considering the wall lattice and one $\mathrm{LJ}$ atom at different locations. The location of the top wall layer is at $y=0 \sigma$. The difference between maxima and minima varies as the distance between the plane that contains the $\mathrm{LJ}$ atom and the top wall layer. (b) The energy barrier $\Delta E$ versus $y$ for $\varepsilon_{\mathrm{WF}}=0.5 \varepsilon$ and $\varepsilon_{\mathrm{WF}}=0.005 \varepsilon$. $\Delta E$ is defined as the difference between maximum (position $\mathrm{A}$ ) and minimum (position B) along the black dashed line shown in the panel (a). hop across the energy barrier between potential minima. The slip velocity originates from the difference between hopping rates in the flow direction and opposite to the flow direction. The slip velocity $\left(v_{\mathrm{S}}\right)$ can be expressed as ${ }^{25}$

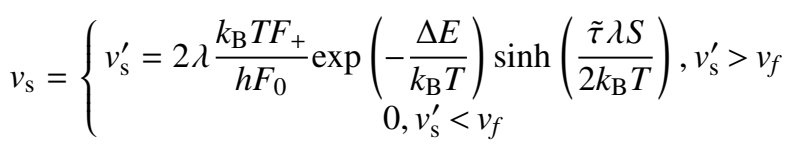

where $T$ is the temperature and $h$ is the Planck constant. $\Delta E$ is the energy barrier that atoms encounter. $F_{+}$and $F_{0}$ are the partition functions for the activated and initial states, respectively. Here, $\tilde{\tau}$ is the shear stress acting on FFL, $S$ is the effective area of FFL along the flow direction, and $\lambda$ is the distance of a single hop in the FFL. $v_{f}$ is the typical fluctuation of thermal velocity in the FFL. The apparent slip velocity in Eq. (2) becomes finite when $v_{S}^{\prime}$ can be discerned from the fluctuation of thermal velocity; otherwise, the shear stress only enhances the thermal motion of liquid atoms. ${ }^{25}$ At low shear rates, the term $\tilde{\tau} \lambda S$ is much smaller than $k_{\mathrm{B}} T$, and thus the hyperbolic sine function in Eq. (2) can be reduced to the linear function and $F_{+} / F_{0}$ can be assumed to be constant. Therefore, Eq. (2) for the average slip velocity reduces to

$$
v_{\mathrm{s}} \propto \tilde{\tau} \lambda^{2} S \exp \left(-\frac{\Delta E}{k_{\mathrm{B}} T}\right) .
$$

In general, the energy barrier $\Delta E$ depends on $\varepsilon_{\mathrm{WF}}$, the distance from the top lattice plane, and the wall lattice constant.
In the present study, we focused on the first two factors. As it is evident from Fig. 4(b), $\Delta E$ increases sharply as the distance between the $\mathrm{LJ}$ atom and lattice plane decreases. For the same distance from the top lattice plane, $\Delta E$ decreases at small $\varepsilon_{\mathrm{WF}}$ and the sharply increasing part of $\Delta E-y$ curve is displaced closer to the wall at the small $\varepsilon_{\mathrm{WF}}$.

The fluid density profiles near the lower wall are presented in Fig. 5 for the indicated system parameters. It can be seen in Fig. 5(a) that the density distribution of atoms in the FFL is markedly different at large and small values of $\varepsilon_{\mathrm{WF}}$. At large $\varepsilon_{\mathrm{WF}}$, the location of the FFL (measured by the position of the first density maximum) remains nearly the same and the first peak is relatively sharp [see Fig. 5(a)]. These features of the density profiles of fluid atoms in the FFL support the validity of assumption that $\Delta E$ is constant in Eqs. (2) and (3) for large enough $\varepsilon_{\mathrm{WF}}$. In other words, $\Delta E$ depends on the distance from the top lattice plane in an insignificant way.

When $\varepsilon_{\mathrm{WF}}$ decreases below a certain value, the location of FFL is displaced towards the wall, and fluid atoms become more broadly distributed within the FFL [see Figs. 5(a) and 5(b2)]. We note, however, that fluid atoms do not penetrate into the solid wall even at the smallest value $\varepsilon_{\mathrm{WF}}=0.0035 \varepsilon$ considered in the present study. The broader density distribution within the FFL suggests that the equal energy barrier assumption might not be valid at small $\varepsilon_{\mathrm{WF}}$ since atoms at different positions experience an energy barrier of different magnitudes. In this case, the dependence of $\Delta E$ on the location
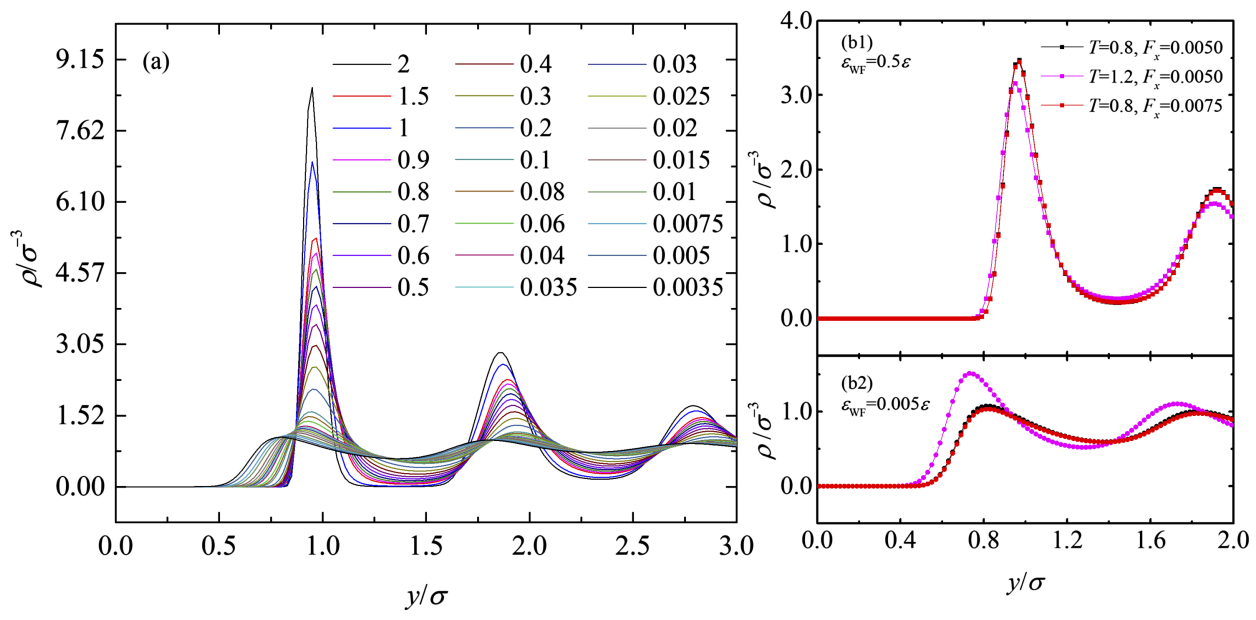

FIG. 5. (a) The fluid density profiles perpendicular to the lower wall for $\varepsilon_{\mathrm{WF}}$ in the range from $2.0 \varepsilon$ to $0.0035 \varepsilon$ at $T=0.8$ and $F_{x}=0.005$. The units of $T$ and $F_{x}$ are $\varepsilon / k_{\mathrm{B}}$ and $\varepsilon / \sigma$. (b) The fluid density profiles perpendicular to the wall for the indicated system parameters. 
of FFL should be taken into account. To consider the dependence of $\Delta E$ on the position of atoms within FFL and $\varepsilon_{\mathrm{WF}}$, we introduce the density-weighted average energy barrier, $\overline{\Delta E}$,

$$
\overline{\Delta E}=\frac{\sum_{i=1}^{n} \rho_{i} \Delta E_{i}}{\sum_{i=1}^{n} \rho_{i}},
$$

where $\rho_{i}$ is the density in the $i$ th slice parallel to the lattice plane, $\Delta E_{i}$ is the energy barrier for atoms in the $i$ th slice, and $n$ is the total number of slices in the FFL.

The dependence of the density-averaged energy barrier $\overline{\Delta E}$ as a function of $\varepsilon_{\mathrm{WF}}$ for the indicated parameters is presented in Fig. 6(a). In all cases, $\overline{\Delta E}$ first decreases and then increases with $\varepsilon_{\mathrm{WF}}$ decreasing. We then calculated the factor $\exp \left(-\overline{\Delta E} /\left(k_{\mathrm{B}} T\right)\right)$ using the corresponding values of $\overline{\Delta E}$ at different $\varepsilon_{\mathrm{WF}}$ for cases at a given $T$ and $F_{x}$. The Boltzmann constant $\left(k_{\mathrm{B}}\right)$ is set to 1 for the unit system used in our study. The values of temperature used to calculated $\exp \left(-\overline{\Delta E} /\left(k_{\mathrm{B}} T\right)\right)$ are in the range from $0.8 \varepsilon / k_{\mathrm{B}}$ to $1.6 \varepsilon / k_{\mathrm{B}}$. We directly used the temperature set by the thermostat because the difference between the time-averaged temperature from simulations and the thermostat temperature is negligible. Therefore, the value of $\varepsilon_{\mathrm{WF}}$ at which $\overline{\Delta E}$ is minimum is the same as the value of $\varepsilon_{\mathrm{WF}}$ at which $\exp \left(-\overline{\Delta E} /\left(k_{\mathrm{B}} T\right)\right)$ is maximum for a given $T$ and $F_{x}$. It can be seen in Fig. 6(b) that the critical value of $\varepsilon_{\mathrm{wF}}$ where $\exp \left(-\overline{\Delta E} /\left(k_{\mathrm{B}} T\right)\right)$ reaches maximum or $\overline{\Delta E}$ reaches minimum correlates well with $\varepsilon_{L \mathrm{~s}}^{\max }$.

The non-monotonic dependence of $\overline{\Delta E}$ on $\varepsilon_{\mathrm{WF}}$ is a net result of decreasing $\varepsilon_{\mathrm{WF}}$ and the broader density distribution of the FFL at smaller $\varepsilon_{\mathrm{WF}}$. As mentioned above, $\Delta E$ depends on $\varepsilon_{\mathrm{WF}}$ and the distance from the top lattice plane for a given wall structure. For large $\varepsilon_{\mathrm{wF}}$, the location of FFL remains approximatively unchanged and atoms in the FFL are distributed within a narrow region. Under these circumstances, $\varepsilon_{\mathrm{WF}}$ dominates the magnitude of $\overline{\Delta E}$ resulting in that $\overline{\Delta E}$ decreases with reducing $\varepsilon_{\mathrm{WF}}$. By contrast, for small $\varepsilon_{\mathrm{WF}}$, the location of FFL is displaced towards the wall, and fluid atoms become more broadly distributed within the FFL as $\varepsilon_{\mathrm{WF}}$ decreases. Since $\Delta E$ increases sharply as the distance from the top lattice plane is decreasing, these variations of density distribution of atoms in the FFL dominate $\overline{\Delta E}$ in Eq. (4). Therefore, $\overline{\Delta E}$ increases as $\varepsilon_{\mathrm{WF}}$ decreases for small $\varepsilon_{\mathrm{WF}}$.

Smaller values of $\overline{\Delta E}$ indicate that fluid atoms in the FFL hop more frequently between minima of the surface potential. Hence, slip increases as $\varepsilon_{\mathrm{WF}}$ decreasing. Similarly, after $\varepsilon_{\mathrm{WF}}$ decreases below a certain value, increasing $\overline{\Delta E}$ leads to smaller hopping rate of fluid atoms in the FFL, and, thus, smaller slip. Therefore, the non-monotonic dependence of the slip length on $\varepsilon_{\mathrm{WF}}$ is recovered.

The influence of $T$ on slip is more complicated. On the one hand, at high $T$ atoms in the FFL possess a greater kinetic energy and overcome energy barriers more easily, hence larger slip. On the other hand, atoms in the FFL are displaced towards the wall with increasing $T$ at the same $\varepsilon_{\mathrm{WF}}$ [see Figs. 5(b1) and 5(b2)]. In other words, $\overline{\Delta E}$ increases with $T$, hence smaller slip. Therefore, the dependence of slip on $T$ is a competition between the increase of the energy barrier and kinetic energy of atoms with increasing $T$.

The simulation results for the factor $\exp \left(-\overline{\Delta E} /\left(k_{\mathrm{B}} T\right)\right)$ versus $\varepsilon_{\mathrm{WF}}$ for different $T$ are plotted in Fig. 6(c). At large $\varepsilon_{\mathrm{WF}}$, $T$ has insignificant effect on the density distribution in the
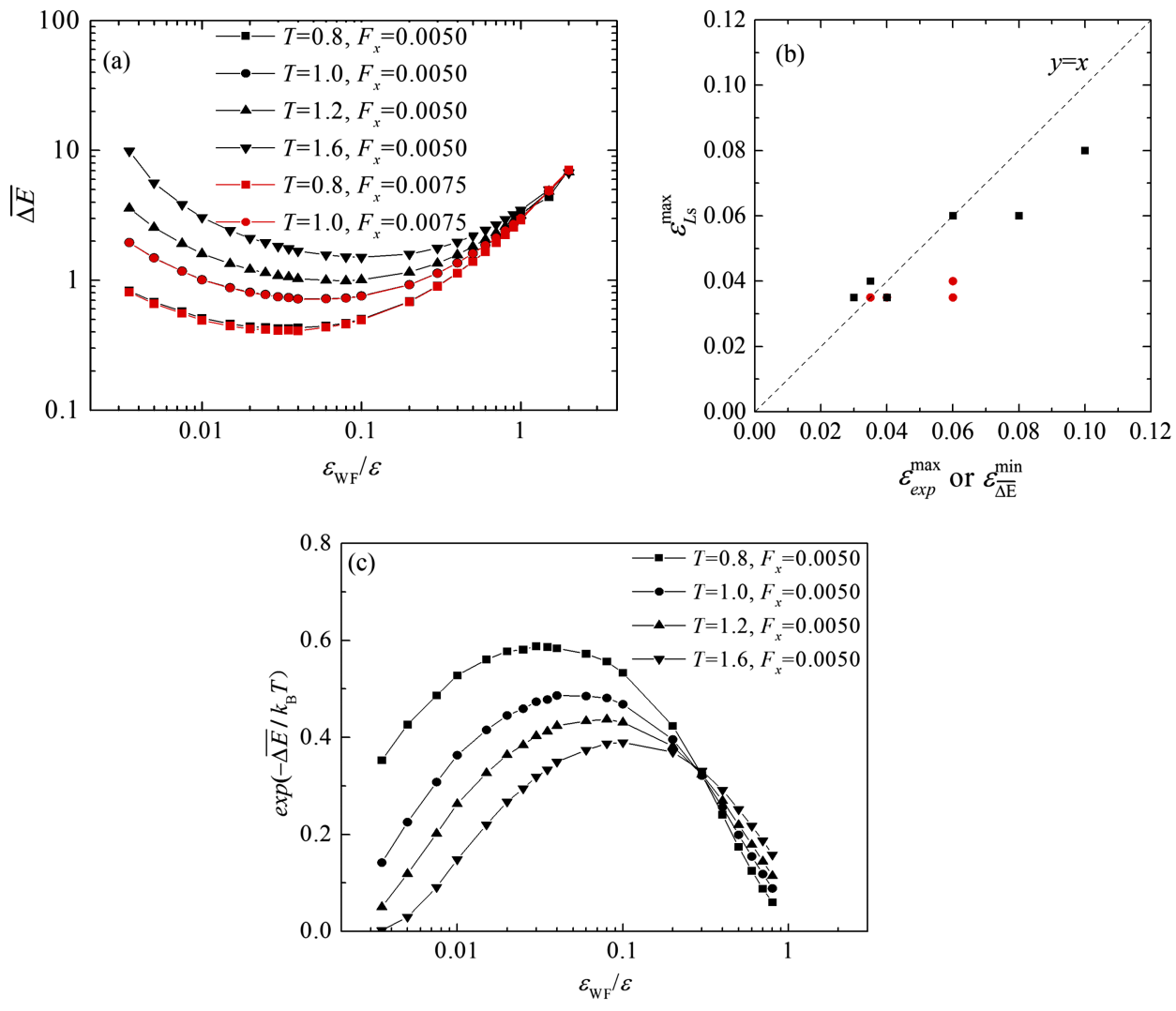

FIG. 6. (a) The density-weighted average energy barrier $(\overline{\Delta E})$ in the FFL versus $\varepsilon_{\mathrm{WF}}$ at different conditions. (b) A correlation $\varepsilon_{L \mathrm{~s}}^{\max }$ versus $\varepsilon_{\mathrm{exp}}^{\max }$ or $\varepsilon_{\overline{\Delta E}}^{\min }$ at different $T$ and $F_{x} . \varepsilon_{L s}^{\max }$ is the critical $\varepsilon_{\mathrm{WF}}$ where $L_{\mathrm{S}}$ reaches maximum. $\varepsilon_{\mathrm{exp}}^{\max }$ and $\varepsilon_{\frac{\mathrm{mE}}{\mathrm{min}}}$ are the critical $\varepsilon_{\mathrm{WF}}$ where $\exp \left(-\frac{\Delta E}{\Delta E} /\left(k_{\mathrm{B}} T\right)\right)$ reaches maximum and $\overline{\Delta E}$ reaches minimum, respectively. Symbols (black filled square) and (red filled circle) denote the data as $T$ varies from $0.8 \varepsilon / k_{\mathrm{B}}$ to $1.6 \varepsilon / k_{\mathrm{B}}$ at $F_{x}$ $=0.005 \varepsilon / \sigma$ and $F_{x}$ varies from $0.0025 \varepsilon / \sigma$ to $0.01 \varepsilon / \sigma$ at $T=1.0 \varepsilon / k_{\mathrm{B}}$, respectively. (c) The quantity, $\exp \left(-\overline{\Delta E} /\left(k_{\mathrm{B}} T\right)\right)$, versus $\varepsilon_{\mathrm{WF}}$ for the selected parameters. 
FFL [see Fig. 5(b1)] owing to the strong WFI. In this case, the kinetic energy $\left(k_{\mathrm{B}} T\right)$ mostly contributes to changes of $\exp \left(-\overline{\Delta E} /\left(k_{\mathrm{B}} T\right)\right)$, shown in Fig. 6(c), leading to larger slip at higher $T$. At small $\varepsilon_{\mathrm{wF}}$, the FFL moves significantly closer to the wall with increasing $T$ [see Fig. 5(b)], which results in a considerable increment of $\overline{\Delta E}$. Consequently, $\overline{\Delta E}$ dominates the factor $\exp \left(-\overline{\Delta E} /\left(k_{\mathrm{B}} T\right)\right)$ [see Fig. 6(c)], thus showing that increasing $T$ decreases slip. We conclude that the positive correlation between $\varepsilon_{L \mathrm{~s}}^{\max }$ and $T$ shown in Fig. 3 originates from the fact that the FFL is displaced towards the wall at larger $\varepsilon_{\mathrm{wF}}$ and higher $T$.

It can be noticed that the FFL is displaced closer towards the walls and the density profile in the FFL becomes sharper with increasing $T$ at small $\varepsilon_{\mathrm{wF}}$ [see Fig. 5(b2)]. As discussed above, $\overline{\Delta E}$ increases as the FFL is displaced closer towards the walls and fluid atoms become more broadly distributed within the FFL. Therefore, with increasing $T$ at small $\varepsilon_{\mathrm{wF}}$, the variation of $\overline{\Delta E}$ is a combined effect of FFL towards the walls and the shaper density profile in the FFL. This phenomenon underlines the advantage of using $\overline{\Delta E}$ to quantify the energy barrier, since $\overline{\Delta E}$ includes both the position of the FFL and features of the fluid density profile in the FFL. It can be observed that there is a difference between the values of $\varepsilon_{\mathrm{CR}}^{\mathrm{I}}$ of intersection in Figs. 2 and 6(c). Also, the trends of curves in Fig. 2 are different from the corresponding ones in Fig. 6(c). These two mismatches might be attributed to the missing factor of shear stress in the term $\exp \left(-\overline{\Delta E} /\left(k_{\mathrm{B}} T\right)\right)$.

At $T=0.8$ and $1.0 \varepsilon / k_{\mathrm{B}}$, the magnitude of $\overline{\Delta E}$ in Fig. 6(a) remains nearly identical when $F_{x}$ is varied from $0.005 \varepsilon / \sigma$ to $0.0075 \varepsilon / \sigma$. At low shear rates, $F_{x}$ has negligible influence on the density distribution of the adjacent fluid at large and small values of $\varepsilon_{\mathrm{WF}}$ [see Figs. 5(b1) and 5(b2)], which is consistent with the previous results. ${ }^{17,20}$ At other conditions remaining the same, increasing driving force leads to a larger slip velocity which is described by the term in front of the exponential factor in Eq. (3). The increase of the slip length with driving force shows that the slip length is rate-dependent in our cases. As discussed above, the critical value $\varepsilon_{L S}^{\max }$ is determined by the exponential factor of $\overline{\Delta E}$. Thus, $\varepsilon_{L \mathrm{~s}}^{\max }$ remains unchanged with $F_{x}$ since driving force influences the term in front of the exponential factor in Eq. (3) at low shear rates.

We finally comment that the fluid density in the bulk changes slightly (the maximum deviation of bulk fluid density is less than $5 \%$ from the expected value of $0.813 \sigma^{-3}$ ), since the effective height of the channel depends on $\varepsilon_{\mathrm{WF}}$ (see Fig. 5), which in turn might affect the fluid viscosity and the slip length. In our study, the bulk viscosity was computed using the following equation:

$$
\mu=\frac{F_{x} \Delta y A \sum_{i=1}^{n} \rho_{i}}{\left(\dot{\gamma}_{1}-\dot{\gamma}_{n}\right) A},
$$

where $\Delta y$ is the thickness of the slice parallel to the lattice plane, $A$ is the area of simulation domain in the $x z$ plane. Indices $i=1$ and $n$ correspond to the $y$-coordinates, $y_{1}$ and $y_{n}\left(y_{n}>y_{1}\right)$. $\dot{\gamma}_{1}$ and $\dot{\gamma}_{n}$ are the shear rates of the fluid phase at $y_{1}$ and $y_{n}$, respectively. $y_{1}$ and $y_{n}$ are located in the bulk region and $y_{n}$ is less than half of the channel height. In our work, $y_{1}$ and $y_{n}$ equals to $7.01 \sigma$ and $14.01 \sigma$.

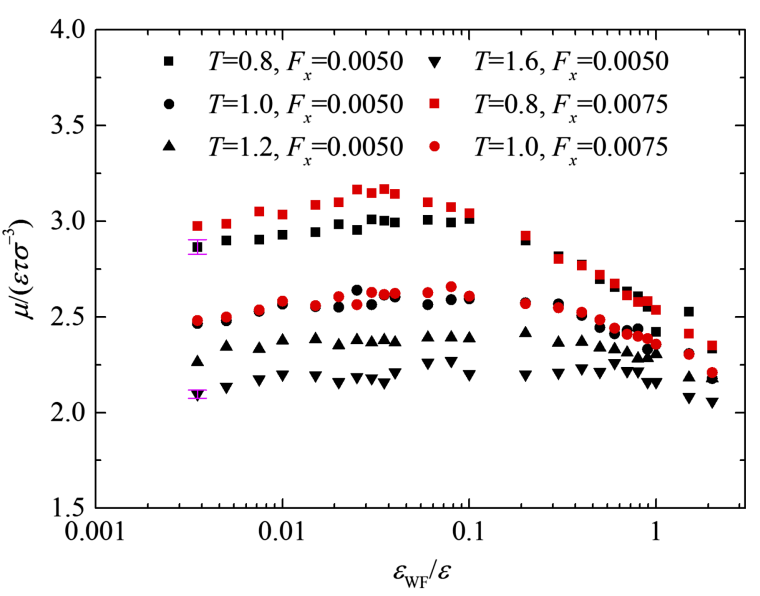

FIG. 7. The fluid viscosity $(\mu)$ versus the wall-fluid interaction energy $\varepsilon_{\mathrm{WF}}$ for different temperatures $(T)$ and driving forces $\left(F_{x}\right)$. The units of $T$ and $F_{x}$ are $\varepsilon / k_{\mathrm{B}}$ and $\varepsilon / \sigma$, respectively. The error bars were estimated from five independent runs.

The relationship between $\mu$ and $\varepsilon_{\mathrm{wF}}$ at different $T$ and $F_{x}$ is presented in Fig. 7. The value of $\mu$ computed using Eq. (5) is consistent with the previous results. ${ }^{18}$ It can be seen in Fig. 7 that the bulk viscosity is nearly constant in the weak WTI regime $\left(\varepsilon_{\mathrm{WF}}<0.1 \varepsilon\right)$ and it varies slightly at large $\varepsilon_{\mathrm{WF}}$ when fluid and solid structures are locked together at interfaces. We note that the variation of the friction coefficient $\left(k=\mu / L_{S}\right)$ as a function of $\varepsilon_{\mathrm{WF}}$ displays the same strong- and weak-WFI slip regimes (as in Fig. 2) and the critical value of $\varepsilon_{\mathrm{WF}}$ is identical to the one reported in Fig. 6(b) (not shown).

\section{SUMMARY}

In this paper, the slip behavior of a simple fluid was investigated in a wide range of the wall-fluid interaction (WFI) energies for force-driven flows using non-equilibrium molecular dynamics simulations. Two slip regimes were observed: the strong-WFI and weak-WFI regimes. In the strong-WFI regime, the slip length increases with WFI decreasing, while slip depends on WFI in an opposite way in the weak-WFI regime. The critical value of the WFI energy when the slip length is maximum increases with temperature but remains unchanged with driving force at low shear rates. Interestingly, we observed that $L_{\mathrm{S}}-\varepsilon_{\mathrm{WF}}$ curves intersect each other at different temperatures. However, the physical meaning of such an intersection point is at present not clear and it will be addressed in our future study.

Furthermore, we demonstrated that the variation of the slip length can be understood by examining the density-weighted average energy barrier $(\overline{\Delta E})$ encountered by fluid atoms in the first fluid layer during their hopping between minima of the surface potential. The relationship between slip and WFI is a combined effect of the reduced WFI energy and different density distributions of FFL on $\overline{\Delta E}$. At the same WFI and driving force, temperature affects $\overline{\Delta E}$ by influencing the density distribution of fluid in the FFL and kinetic energy of atoms. At different WFIs and temperatures, the slip length is well correlated with the exponential factor $\exp \left(-\overline{\Delta E} /\left(k_{\mathrm{B}} T\right)\right)$, which determines the critical value of WFI. 


\section{ACKNOWLEDGMENTS}

This work was supported by the National Science Foundation of China under Grant No. 51679203; the Natural Science Basic Research Plan in Shaanxi Province of China under Grant No. 2016JM1002 and the Fundamental Research Funds for the Central Universities under Grant No. 3102015ZY017.

${ }^{1}$ Y. X. Zhu and S. Granick, Phys. Rev. Lett. 87, 096105 (2001).

${ }^{2}$ R. Pit, H. Hervet, and L. Leger, Phys. Rev. Lett. 85, 980 (2000).

${ }^{3}$ C. Neto, D. R. Evans, E. Bonaccurso, H. J. Butt, and V. S. J. Craig, Rep. Prog. Phys. 68, 2859 (2005).

${ }^{4}$ L. Léger, J. Phys.: Condens. Matter 15, S19 (2003).

${ }^{5}$ E. Bonaccurso, M. Kappl, and H. J. Butt, Phys. Rev. Lett. 88, 076103 (2002).

${ }^{6}$ N. V. Priezjev and S. M. Troian, Phys. Rev. Lett. 92, 018302 (2004).

${ }^{7}$ D. M. Huang, C. Sendner, D. Horinek, R. R. Netz, and L. Bocquet, Phys. Rev. Lett. 101, 226101 (2008).

${ }^{8}$ J. L. Barrat and L. Bocquet, Phys. Rev. Lett. 82, 4671 (1999).

${ }^{9}$ P. A. Thompson and S. M. Troian, Nature 389, 360 (1997).

${ }^{10}$ P. A. Thompson and M. O. Robbins, Phys. Rev. A 41, 6830 (1990).

${ }^{11}$ A. Martini, H.-Y. Hsu, N. A. Patankar, and S. Lichter, Phys. Rev. Lett. 100, 206001 (2008).

${ }^{12}$ B. J. Kirby, Micro- and Nanoscale Fluid Mechanics: Transport in Microfluidic Devices (Cambridge University Press, Cambridge, 2010).
${ }^{13}$ A. Martini, A. Roxin, R. Q. Snurr, Q. Wang, and S. Lichter, J. Fluid Mech. 600, 257 (2008).

${ }^{14}$ J. L. Barrat and L. Bocquet, Faraday Discuss. 112, 119 (1999).

${ }^{15}$ S. Lichter, A. Roxin, and S. Mandre, Phys. Rev. Lett. 93, 086001 (2004).

${ }^{16}$ S. Lichter, A. Martini, R. Q. Snurr, and Q. Wang, Phys. Rev. Lett. 98, 226001 (2007).

${ }^{17}$ N. V. Priezjev, J. Chem. Phys. 127, 144708 (2007).

${ }^{18}$ N. V. Priezjev, Phys. Rev. E 75, 051605 (2007).

${ }^{19}$ N. V. Priezjev, Phys. Rev. E 82, 051603 (2010).

${ }^{20}$ X. Yong and L. T. Zhang, Phys. Rev. E 82, 056313 (2010).

${ }^{21}$ N. Asproulis and D. Drikakis, Phys. Rev. E 84, 031504 (2011).

${ }^{22}$ A. A. Pahlavan and J. B. Freund, Phys. Rev. E 83, 021602 (2011).

${ }^{23}$ X. Yong and L. T. Zhang, Phys. Rev. E 85, 051202 (2012).

${ }^{24}$ L. Bao, H. Hu, J. Wen, P. Sepri, and K. Luo, Sci. Rep. 6, 29786 (2016).

${ }^{25}$ F. C. Wang and Y. P. Zhao, Soft Matter 7, 8628-8634 (2011).

${ }^{26}$ C. Sendner, D. Horinek, L. Bocquet, and R. R. Netz, Langmuir 25, 10768 (2009).

${ }^{27}$ R. S. Voronov, D. V. Papavassiliou, and L. L. Lee, J. Chem. Phys. 124, 204701 (2006).

${ }^{28}$ R. S. Voronov, D. V. Papavassiliou, and L. L. Lee, Chem. Phys. Lett. 441, 273 (2007).

${ }^{29}$ C. Liu and Z. G. Li, J. Chem. Phys. 132, 024507 (2010).

${ }^{30}$ X. Yong and L. T. Zhang, J. Chem. Phys. 138, 084503 (2013).

${ }^{31}$ S. Plimpton, J. Comput. Phys. 117, 1 (1995).

${ }^{32}$ X. Yong and L. T. Zhang, Microfluid. Nanofluid. 14, 299 (2013). 